\title{
On the Positive Definite Solutions of the Matrix Equation $X^{S}+A^{*} X^{-S} A=Q$
}

\author{
Maria Adam*
}

Department of Computer Science and Biomedical Informatics, University of Central Greece, Lamia 35100, Greece

\begin{abstract}
In this paper, necessary and sufficient conditions for the existence of the positive definite solutions of the nonlinear matrix equation $X^{s}+A^{*} X^{-s} A=Q$ are presented, when $A$ is nonsingular and $s$ an integer, as well as some new properties and bounds for the eigenvalues of matrices related to $A, Q$ are discussed. An algebraic method for the computation of the solutions is proposed, based on the algebraic solution of the corresponding discrete time Riccati equation. The exact number of the positive definite solutions is also computed.
\end{abstract}

Mathematics Subject Classification: 15A60, 15A18, 15A24, 93B40.

Keywords: Numerical radius, Eigenvalue, Riccati equation.

\section{INTRODUCTION}

The central issue of this paper is the investigation and the computation of the positive definite solutions of the matrix equation

$X^{s}+A^{*} X^{-s} A=Q$,

where the nonsingular matrix $A \in M_{n}, M_{n}$ denotes the set of all $n \times n$ matrices with complex or real entries, $A^{*}$ stands for the conjugate transpose of $A, Q \in M_{n}$ is a positive definite matrix and $s$ is an integer. The matrix equation of the form (1) arises in many applications in a wide variety of research areas including control theory, ladder networks, dynamic programming, stochastic filtering and statistics, see [1-3] and the references given therein. In the case that $A$ is nonsingular and $s=1$, necessary and sufficient conditions for the existence of a positive definite solution of the matrix equation (1) have been investigated by many authors $[1,2,4-6]$ and formulas for the computation of Hermitian and non-Hermitian solutions can be found in [4].

The more general equation $X^{s}+A^{*} X^{-t} A=Q$, with $A$ nonsingular, has been considered in [7-9] (see also references therein), when $s, t$ are positive integers and in [10] when $s \geq 1,0<t \leq 1$ and $0<s \leq 1, t \geq 1$; there some existence conditions and properties of its positive definite solutions are obtained. Also, in some papers numerical methods for computing the extreme solutions and several effective iterative algorithms have been proposed [9-12] and a perturbation analysis of the positive definite solutions has been presented.

To describe our results, we introduce some notations and definitions. For $A, B \in M_{n}$, the notation $A>B(A \geq B)$

*Address correspondence to this author at the Department of Computer Science and Biomedical Informatics, University of Central Greece, Lamia 35100, Greece; Tel: +30 22310 66900; Fax: +30-22310-66939;

E-mail:madam@ucg.gr means that $A$ and $B$ are Hermitian matrices and $A-B$ is a positive definite (semidefinite) matrix, which is denoted by $A-B>0(A-B \geq 0)$. For $A \in M_{n}, \quad \lambda_{i}(A)$ denotes an eigenvalue of $A, \sigma(A)$ the spectrum of $A$,

$$
\begin{aligned}
& \rho(A)=\max \left\{\left|\lambda_{i}(A)\right|: \lambda_{i}(A) \in \sigma(A)\right\} \\
& \|A\|=\max \left\{\sqrt{\lambda_{i}\left(A^{*} A\right)}: \lambda_{i}\left(A^{*} A\right) \in \sigma\left(A^{*} A\right)\right\}
\end{aligned}
$$

denotes the spectral radius and spectral norm of $A$, respectively, and

$r(A)=\max \left\{\left|x^{*} A x\right|\right.$ : for each vector $x \in \mathbb{C}^{n}$, with $\left.x^{*} x=1\right\}(2)$

the numerical radius of $A$. Let $A \in M_{n}$ be a Hermitian matrix, $\quad \lambda_{i}(A) \in \sigma(A)$ are indexed in increasing order $\lambda_{\text {min }}(A)=\lambda_{1}(A) \leq \lambda_{2}(A) \leq \cdots \leq \lambda_{n}(A)=\lambda_{\max }(A)$. It is also known [1,3-6] that, when the matrix equation (1) for $s=1$ has a positive definite solution $X$, then there exist minimal and maximal solutions $X_{\min }$ and $X_{\max }$, respectively, such that $0<X_{\min } \leq X \leq X_{\max }$ for any positive definite solution $X$. The minimal and maximal solutions are referred as the extreme solutions. Moreover, the existence of a positive definite solution depends on the numerical radius of the matrix $Q^{-1 / 2} A Q^{-1 / 2}$, [5, Theorem 5.2].

Note that, setting in (1)

$Y=X^{s}$

we obtain

$Y+A^{*} Y^{-1} A=Q$.

Furthermore without loss of generality, $s$ in (1) may be assumed to be a positive integer. Indeed, in the case that $s \leq-1$ setting $Z=X^{-1}$ the equation (1) takes the form

$Z^{-s}+A^{*} Z^{s} A=Q \Rightarrow Z^{s_{1}}+A^{*} Z^{-s_{1}} A=Q$, for $s_{1} \geq 1$. 
In this paper, we focus on the computation of the positive definite solutions of the matrix equation (1), with $A \in M_{n}$ a nonsingular matrix, $Q>0$ and $s$ an integer with $s \geq 1$. In Section 2, necessary and sufficient conditions for the existence of positive definite solutions of (1) are obtained, a new inequality for the spectral radius of $A, Q$ is presented and in Theorem 7 a formula for computing all the positive definite solutions of (1) is given; when the matrices $A, Q$ have special properties, more specific necessary and sufficient conditions for the existence of solutions of (1) are discussed, related to some new inequalities for their eigenvalues. In Section 3, bounds for the eigenvalues of the minimal solution of (1) are given; an algebraic method for computing the positive definite solutions is proposed, based on the algebraic solution of the corresponding discrete time Riccati equation and the exact number of positive definite solutions of (1) is computed, when these exist. In Section 4, a numerical result is given to illustrate the efficiency of the proposed method and finally, concluding remarks are given in Section 5.

\section{EXISTENCE AND FORMULA OF THE POSITIVE DEFINITE SOLUTIONS OF $X^{s}+A^{*} X^{-s} A=Q$}

In the first part of this section, we utilize the concept of numerical radius in order to derive necessary and sufficient conditions for the existence of positive definite solutions of (1). We need the following lemma.

Lemma 1 [13, Theorem 7.2.6] Let $A \in M_{n}$ be a positive semidefinite matrix and let $s \geq 1$ be a given integer. Then there exists a unique positive semidefinite matrix $B$ such that $B^{s}=A$.

Theorem 2 Let $A \in M_{n}$ be a nonsingular matrix and $Q \in M_{n}$ with $Q>0$. The equation $X^{s}+A^{*} X^{-s} A=Q$ has a positive definite solution $X \in M_{n}$ if and only if for the numerical radius of $Q^{-1 / 2} A Q^{-1 / 2}$ holds

$r\left(Q^{-1 / 2} A Q^{-1 / 2}\right) \leq \frac{1}{2}$.

Proof. Since the solving of the matrix equation (1) is equivalent to (4) by (3), according to Lemma 1 assuming that $Y \in M_{n}$ is a positive definite solution of (4), then there exists a unique $s$ th root of $Y \in M_{n}$, which is $X>0$ and solves the equation (1). Hence, it is needed the proof of the equivalence between the numerical radius in (5) and the solving of (4), which follows essentially the same lines as the proof of Theorem 5.2 of Engwerda et al. [5] and is omitted.

Remark 1 Consider that for the matrices $A, Q \in M_{n}$ of the nonlinear matrix equation (1) holds $r\left(Q^{-1 / 2} A Q^{-1 / 2}\right) \leq \frac{1}{2}$. According to Theorem 2 and [8, Theorem 3.3] it is easy to be proved that for the eigenvalues of $A^{*} A, Q \in M_{n}$, the following inequality holds: $\lambda_{\text {min }}\left(A^{*} A\right) \leq \frac{1}{4}(\rho(Q))^{2}$

The following result presents the relation of spectral radii of $A, Q$ in (1); the conclusion is the same as in [7, Theorem 3.1] without the assumption that the matrices satisfy $A Q^{1 / 2}=Q^{1 / 2} A$.

Theorem 3 Let $A \in M_{n}$ be a nonsingular matrix, $Q \in M_{n}$ with $Q>0$. If the matrix equation $X^{s}+A^{*} X^{-s} A=Q$ has a positive definite solution, then

$\rho(A) \leq \frac{1}{2} \rho(Q)$.

Proof. Let $\lambda(A) \in \sigma(A)$ and $x$ its corresponding eigenvector. Multiplying the equation (1) on the left and on the right by $x^{*}$ and $x$ respectively, due to $A x=\lambda(A) x$, we take :

$$
\begin{aligned}
& x^{*} X^{s} x+x^{*} A^{*} X^{-s} A x=x^{*} Q x \\
& x^{*} X^{s} x+|\lambda(A)|^{2} x^{*} X^{-s} x=x^{*} Q x
\end{aligned}
$$

Since $X>0$, there exists a unitary matrix $V \in M_{n}$ and a positive real diagonal matrix $D=\operatorname{diag}\left\{\lambda_{1}(X), \cdots, \lambda_{n}(X)\right\}$, $\lambda_{i}(X) \in \sigma(X),(1 \leq i \leq n)$, such that $X=V D V^{*}$. Using the above notation, the equation (7) can be rewritten as

$x^{*} V D^{s} V^{*} x+|\lambda(A)|^{2} x^{*} V D^{-s} V^{*} x=x^{*} Q x$.

Setting the nonzero vector $y=V^{*} x \in \mathbb{C}^{n}$ we have

$y^{*} D^{s} y+|\lambda(A)|^{2} y^{*} D^{-s} y=y^{*} V^{*} Q V y$,

which implies

$$
\begin{aligned}
|\lambda(A)|^{2} & =\frac{y^{*} V^{*} Q V y-y^{*} D^{s} y}{y^{*} D^{-s} y} \leq \frac{\lambda_{\max }(Q) y^{*} y-y^{*} D^{s} y}{y^{*} D^{-s} y} \\
& =\frac{\sum_{i=1}^{n}\left|y_{i}\right|^{2}\left(\lambda_{\max }(Q)-\lambda_{i}^{s}(X)\right)}{\sum_{i=1}^{n}\left|y_{i}\right|^{2} \lambda_{i}^{-s}(X)} .
\end{aligned}
$$

Consider the function $f(t)=t^{s}\left(\lambda_{\max }(Q)-t^{s}\right)$, with $t \in[0,+\infty)$. It is monotonically increasing on $\left[0,\left(\frac{\lambda_{\max }(Q)}{2}\right)^{1 / s}\right]$, and monotonically decreasing on $\left[\left(\frac{\lambda_{\max }(Q)}{2}\right)^{1 / s},+\infty\right)$, and $\left.f_{\max }=f\left(\frac{\lambda_{\max }(Q)}{2}\right)^{1 / s}\right)=\frac{\lambda_{\max }^{2}(Q)}{4}$, [8, Lemma 3.1]. Thus, for every $t \in[0,+\infty)$ holds

$f(t) \leq \frac{\lambda_{\max }^{2}(Q)}{4} \Rightarrow \lambda_{\max }(Q)-t^{s} \leq \frac{\lambda_{\max }^{2}(Q)}{4 t^{s}}$.

Application of this inequality for $t=\lambda_{i}(X)$, $i=1,2, \ldots, n$, and substitution in (8) yields: 


$$
\begin{aligned}
|\lambda(A)|^{2} & \leq \frac{\sum_{i=1}^{n}\left|y_{i}\right|^{2}\left(\lambda_{\max }(Q)-\lambda_{i}^{s}(X)\right)}{\sum_{i=1}^{n}\left|y_{i}\right|^{2} \lambda_{i}^{-s}(X)} \leq \frac{1}{4} \frac{\sum_{i=1}^{n}\left|y_{i}\right|^{2} \lambda_{\max }^{2}(Q) \lambda_{i}^{-s}(X)}{\sum_{i=1}^{n}\left|y_{i}\right|^{2} \lambda_{i}^{-s}(X)} \\
& =\frac{\lambda_{\max }^{2}(Q)}{4} .
\end{aligned}
$$

Since $Q>0$, clearly denote $\lambda_{\max }(Q)=\rho(Q)$, thus the above inequality yields (6).

In the following, we discuss necessary and sufficient conditions for the existence of solutions of the nonlinear matrix equation (1) utilizing the property of numerical radius, when the matrices $A, Q$ have special properties.

Proposition 4 Let $A \in M_{n}$ be a normal matrix. The equation $X^{s}+A^{*} X^{-s} A=a I, \quad(a>0)$, has a positive definite solution $X \in M_{n}$ if and only if $A$ satisfies one from the following inequalities:

$$
\text { (ii) } \quad \rho(A) \leq \frac{a}{2}
$$

Proof. It is well known [14, p. 45] that for a normal matrix $A$ holds:

$$
r(A)=\rho(A)=\|A\|
$$

By Theorem 2, the existence of a positive definite solution $X \in M_{n}$ of the matrix equation $X^{s}+A^{*} X^{-s} A=a I$ is equivalent to

$$
\begin{aligned}
& r\left((a I)^{-1 / 2} A(a I)^{-1 / 2}\right)=r\left(a^{-1} A\right)=\frac{1}{|a|} r(A) \\
& =\frac{1}{a} r(A) \leq \frac{1}{2} \Leftrightarrow r(A) \leq \frac{a}{2}
\end{aligned}
$$

Hence, combining (10) and (9) arises either (i) or (ii) inequality.

Remark 2 The above Proposition 4 can be viewed as a generalization of [8, Corollary 4.1] and [2, Theorems 11,13], which are special cases of it.

We need the following lemma in order to give a new estimate for the eigenvalues of a normal matrix $A$ and $Q>0$ in (1).

Lemma 5 [8, Lemma 4.2] Let $A \in M_{n}$ be a normal and nonsingular matrix. If $Q \in M_{n}$ is a Hermitian matrix with $A Q=Q A$, then there exist the diagonal matrices $D_{A}, D_{Q}$ and a unitary matrix $V$, such that

$V^{*} A V=D_{A}$ and $V^{*} Q V=D_{Q}$,

that is, $A, Q$ are simultaneously diagonalizable from a unitary matrix $V$.
Proposition 6 Let $A \in M_{n}$ be a normal matrix, $Q \in M_{n}$ with $Q>0$ and $A Q=Q A$. If the equation (1) has a positive definite solution, then

$\max _{1 \leq i \leq n}\left\{\frac{\left|\lambda_{i}(A)\right|}{\lambda_{i}(Q)}, \lambda_{i}(A) \in \sigma(A), \lambda_{i}(Q) \in \sigma(Q)\right\} \leq \frac{1}{2}$.

Proof. Suppose that there exists a unitary matrix $V \in M_{n}$, which simultaneously diagonalizes the matrices $A, Q \quad$ as in Lemma 5, let $A=V D_{A} V^{*}$, with $D_{A}=\operatorname{diag}\left\{\lambda_{1}(A), \ldots, \lambda_{n}(A)\right\}, \quad \lambda_{i}(A) \in \sigma(A) \quad$ and $Q=V D_{Q} V^{*}$, where $D_{Q}=\operatorname{diag}\left\{\lambda_{1}(Q), \ldots, \lambda_{n}(Q)\right\}>0$ due to $\lambda_{i}(Q) \in \sigma(Q)$ are positive real numbers. The diagonal form and the unitary similarity invariance property of numerical radius of the matrices $A, Q$ [14], that is

$$
r(A)=r\left(V D_{A} V^{*}\right) \text { and } r(Q)=r\left(V D_{Q} V^{*}\right),
$$

allow us to write

$$
\begin{aligned}
r\left(Q^{-1 / 2} A Q^{-1 / 2}\right) & =r\left(V D_{Q}^{-1 / 2} V^{*}\left(V D_{A} V^{*}\right) V D_{Q}^{-1 / 2} V^{*}\right) \\
& =r\left(V D_{A} D_{Q}^{-1} V^{*}\right)=r\left(D_{A} D_{Q}^{-1}\right),
\end{aligned}
$$

with $D_{A} D_{Q}^{-1}=\operatorname{diag}\left\{\frac{\lambda_{1}(A)}{\lambda_{1}(Q)}, \ldots, \frac{\lambda_{n}(A)}{\lambda_{n}(Q)}\right\}$. The validity of (11) now follows from (12), the inequality (5) of Theorem 2 and the definition of numerical radius of $D_{A} D_{Q}^{-1}$ by (2).

\section{Remark 3}

1. Assume that the unitary matrix $V$, which simultaneously diagonalizes the matrices $A, Q$ in proof of Proposition 6, gives the diagonal matrices $D_{A}, D_{Q}$ with $\lambda_{i}(A) \in \sigma(A), \lambda_{i}(Q) \in \sigma(Q)$, such that the corresponding eigenvalues are indexed in increasing order $0<\left|\lambda_{\text {min }}(A)\right|=\left|\lambda_{1}(A)\right| \leq\left|\lambda_{2}(A)\right| \leq \cdots$ $\leq\left|\lambda_{n}(A)\right|=\left|\lambda_{\text {max }}(A)\right|=\rho(A)$ and $0<\lambda_{\text {min }}(Q)=\lambda_{1}(Q) \leq$ $\lambda_{2}(Q) \leq \cdots \leq \lambda_{n}(Q)=\lambda_{\max }(Q)=\rho(Q), \quad$ respectively. Since $A$ normal holds (9), thus (11) leads to inequality

$$
\frac{\rho(A)}{\rho(Q)} \leq \frac{1}{2},
$$

i.e., (6) can be written equivalently

$$
\|A\| \leq \frac{1}{2} \rho(Q) \text {. }
$$

Hence, if the matrix equation (1) has a positive definite solution with the matrices $A, Q \in M_{n}$ satisfying the assumptions of Proposition 6 and the unitary matrix $V$ is as above, then, for the largest eigenvalues of $A^{*} A, Q$, the inequality (13) allow us to write 
$\lambda_{\max }\left(A^{*} A\right) \leq \frac{1}{4}(\rho(Q))^{2}$.

Since $\quad A^{*} A>0, \quad$ it follows $0 \leq \lambda_{\text {min }}\left(A^{*} A\right) \leq$ $\lambda_{2}\left(A^{*} A\right) \leq \ldots \leq \lambda_{\max }\left(A^{*} A\right)$ consequently (14) gives an upper bound for $\sigma\left(A^{*} A\right)$.

2. The unitary matrix $V$, which simultaneously diagonalizes $A, Q$, does not in general fulfil the assumptions in the preceding remark as presented in [7, Corollary 3.2] and [8, Theorem 4.1].

3. Note that in the special case that $Q=a I, a>0$, the spectral radius is $\rho(Q)=a$, therefore (13) is exactly (i) or (ii) inequality of Proposition 4. Then (14) is rewritten

$$
\lambda_{\max }\left(A^{*} A\right) \leq \frac{a^{2}}{4}
$$

that the eigenvalues of $A^{*} A$ lie in the interval $\left(0, \frac{a^{2}}{4}\right]$.

In the following theorem, a formula for the positive definite solutions of (1) is given, when the above exist.

Theorem 7 Let $A \in M_{n}$ be a nonsingular matrix, $Q>0$, with $r\left(Q^{-1 / 2} A Q^{-1 / 2}\right) \leq \frac{1}{2}$ and $Y \in M_{n}$ be a positive definite solution of the equation (4). Then for each $Y$ there exist a unitary matrix $U \in M_{n}$, which diagonalizes $Y$, and a unique positive definite solution $X \in M_{n}$ of (1), such that

$X=U \operatorname{diag}\left\{\lambda_{1}^{1 / s}(Y), \lambda_{2}^{1 / s}(Y), \ldots, \lambda_{n}^{1 / s}(Y)\right\} U^{*}$,

where $\lambda_{i}(Y) \in \sigma(Y), i=1,2, \ldots, n$.

Proof. Since $r\left(Q^{-1 / 2} A Q^{-1 / 2}\right) \leq \frac{1}{2}$, according to Theorem 2 the equation (1) has at least one positive definite solution; also, the same condition guarantees the existence at least one positive definite solution of (4) from [5, Theorem 5.2], which is denoted by $Y \in M_{n}$. According to the spectral theorem for $Y$, there exists a unitary matrix $U \in M_{n}$ such that

$Y=U D U^{*}$,

where $D=\operatorname{diag}\left\{\left(\lambda_{1}(Y), \ldots, \lambda_{n}(Y)\right\}\right.$ and $\lambda_{i}(Y) \in \sigma(Y)$ are positive real numbers. Combining the equation in (3) with the above diagonal form of $Y$ in (16), we define the matrix $X \in M_{n}$ as

$X=U D^{1 / s} U^{*}=U \operatorname{diag}\left\{\lambda_{1}^{1 / s}(Y), \ldots, \lambda_{n}^{1 / s}(Y)\right\} U^{*}$,

where the unique positive $s$ th root is well defined in each case due to $\lambda_{i}(Y)>0$. Clearly, using the diagonal forms of $X, Y$ from (15), (16), we derive :

$$
\begin{aligned}
& X^{s}+A^{*} X^{-s} A=U \operatorname{diag}\left\{\lambda_{1}^{1 / s}(Y), \ldots, \lambda_{n}^{1 / s}(Y)\right\}^{s} U^{*} \\
& +A^{*}\left(U^{*}\right)^{-1} \operatorname{diag}\left\{\lambda_{1}^{1 / s}(Y), \ldots, \lambda_{n}^{1 / s}(Y)\right\}^{-s} U^{-1} A \\
& =U D U^{*}+A^{*}\left(U^{*}\right)^{-1} D^{-1} U^{-1} A=Y+A^{*} Y^{-1} A=Q
\end{aligned}
$$

Hence, $X$ consists a solution of (1), which is a positive definite due to $\lambda_{i}(Y)>0$, and unique by Lemma 1 .

\section{RICCATI EQUATION SOLUTION METHOD FOR THE COMPUTATION OF POSITIVE DEFINITE SOLUTIONS OF $X^{S}+A^{*} X^{-S} A=Q$}

Let $r\left(Q^{-1 / 2} A Q^{-1 / 2}\right) \leq \frac{1}{2}$ and $Y$ be a positive definite solution of (4). Working as in [1] and [4], we are able to derive a Riccati equation, which is equivalent to the matrix equation (4). In particular, the matrix equation (4) can be written as

$Y=Q-A^{*} Y^{-1} A$,

whereby the following equivalent equation arises :

$$
\begin{aligned}
Y & =Q-A^{*}\left[Q-A^{*} Y^{-1} A\right]^{-1} A \\
& =Q-A^{*} A^{-1}\left[\left(A^{*}\right)^{-1} Q A^{-1}-Y^{-1}\right]^{-1}\left(A^{*}\right)^{-1} A \\
& =Q+A^{*} A^{-1}\left[Y^{-1}+\left(-\left(A^{*}\right)^{-1} Q A^{-1}\right)\right]^{-1}\left(A^{*} A^{-1}\right)^{*}
\end{aligned}
$$

Substituting in the above equation

$F=A^{*} A^{-1} \quad$ and $\quad G=-\left(A^{*}\right)^{-1} Q A^{-1}$

the related discrete time Riccati equation is derived :

$Y=Q+F\left(Y^{-1}+G\right)^{-1} F^{*}$

Therefore (4) is equivalent to the related discrete time Riccati equation (18), for the solution of which the algebraic Riccati Equation Solution Method can be used [15].

More specifically, from the Riccati equation's parameters in (17), the following matrix is formed

$\Phi=\left[\begin{array}{cc}A^{-1} A^{*} & -A^{-1} Q A^{-1} \\ Q A^{-1} A^{*} & A^{*} A^{-1}-Q A^{-1} Q A^{-1}\end{array}\right]$,

which satisfies $\Phi^{*} J \Phi=J$, where $J=\left[\begin{array}{rr}\mathbb{O} & -I \\ I & \mathbb{O}\end{array}\right]$, i.e., $\Phi$ is a symplectic matrix. All the eigenvalues of $\Phi$ are non-zero $(0 \notin \sigma(\Phi))$ and it may be diagonalized in the form $\Phi=W L W^{-1}$,

where $L$ is a $2 n \times 2 n$ diagonal matrix with diagonal entries the eigenvalues of $\Phi$

$L=\left[\begin{array}{ll}L_{1} & \mathbb{O} \\ \mathbb{O} & L_{2}\end{array}\right]$ 
and $W$ contains the corresponding eigenvectors

$W=\left[\begin{array}{ll}W_{11} & W_{12} \\ W_{21} & W_{22}\end{array}\right]$.

In the case that (4) is solvable, then all its solutions $Y_{j}$, $j=1,2, \ldots, 2 n$ are given by the formulas:

$Y_{j}=W_{21} W_{11}^{-1}$ and $Y_{j}=W_{22} W_{12}^{-1}$,

where the block matrices $W_{11}, W_{12}, W_{21}, W_{22}$ are defined by the partition of $W$ in (21) following every different permutation of its column [4, Propositions 1,2]. Among $Y_{j}$ there exist positive definite solutions for some $j=1,2, \ldots, 2 n$, therefore there exist unitary matrices $U_{j}$, and $D=\operatorname{diag}\left\{\lambda_{1}\left(Y_{j}\right), \ldots, \lambda_{n}\left(Y_{j}\right)\right\} \in M_{n}$, with $\lambda_{i}\left(Y_{j}\right) \in \sigma\left(Y_{j}\right)$ such that

$Y_{j}=U_{j} \operatorname{diag}\left\{\lambda_{1}\left(Y_{j}\right), \ldots, \lambda_{n}\left(Y_{j}\right)\right\} U_{j}^{*}$.

Hence, due to the fact that the assumption of Theorem 7 holds, combining the last relation with (15) each positive definite solution of (1) is given by

$X=U_{j} \operatorname{diag}\left\{\lambda_{1}^{1 / s}\left(Y_{j}\right), \ldots, \lambda_{n}^{1 / s}\left(Y_{j}\right)\right\} U_{j}^{*}$.

We remind that $\Phi$ is a symplectic matrix and its eigenvalues occur in reciprocal pairs. Therefore, we may arrange them in the diagonal matrix $L$ in (20) so that $L_{1}$ to contain all the eigenvalues of $\Phi$, which lie outside the unit circle, and $L_{2}=L_{1}^{-1}$. The above process defines a corresponding (special) arrangement of eigenvectors of $\Phi$ in $W$, which we denote by

$\hat{W}=\left[\begin{array}{ll}\hat{W}_{11} & \hat{W}_{12} \\ \hat{W}_{21} & \hat{W}_{22}\end{array}\right]$.

Using the matrix $\hat{W}$, the unique positive definite solutions of the discrete time Riccati equation in (18) coincide with the extreme solutions of (4) and these are formed :

$Y_{\max }=\hat{W}_{21} \hat{W}_{11}^{-1} \quad$ and $\quad Y_{\min }=\hat{W}_{22} \hat{W}_{12}^{-1}$

Furthermore, it is well known $[1,6]$ that the minimal solution $Y_{\min }$ of (4) and the maximal solution $Z_{\max }$ of the following equation

$Z+A Z^{-1} A^{*}=Q$

satisfy the relation:

$Y_{\min }=Q-Z_{\max }$

Thus from (24), it becomes obvious that the minimal solution of (4) can be derived via the maximal solution of the equation (23), which is of type (4).
Note that if $A$ is Hermitian, then the matrix equations (4) and (23) coincide, consequently from (24) the minimal solution is given

$Y_{\text {min }}=Q-Y_{\text {max }}$.

In the following theorem, bounds for the eigenvalues of the minimal solution of (1) are derived, which are related to the eigenvalues of the maximal solution.

Theorem 8 Let $A \in M_{n}$ be a Hermitian and nonsingular matrix and $Q \in M_{n}$ with $Q>0$. If the equation (1) has a positive definite solution and $\lambda_{\max }^{s}\left(X_{\max }\right) \leq \lambda_{\min }(Q)$, then

$$
\begin{aligned}
& \sqrt[s]{\lambda_{\text {max }}(Q)-\lambda_{\text {max }}^{s}\left(X_{\text {max }}\right)} \leq \lambda_{\text {max }}\left(X_{\text {min }}\right) \leq \sqrt[s]{\lambda_{\text {max }}(Q)-\lambda_{\text {min }}^{s}\left(X_{\text {max }}\right)}, \\
& \sqrt[s]{\lambda_{\text {min }}(Q)-\lambda_{\text {max }}^{s}\left(X_{\text {max }}\right)} \leq \lambda_{\text {min }}\left(X_{\text {min }}\right) \leq \sqrt[s]{\lambda_{\text {min }}(Q)-\lambda_{\text {min }}^{s}\left(X_{\text {max }}\right)},
\end{aligned}
$$

where $X_{\max }, X_{\min }$ are the extreme solutions of (1).

Proof. Since $A$ is Hermitian and (1) has a positive definite solution, it is clear that there exists at least one positive definite solution of (4) as well as the extreme solutions $Y_{\max }$ and $Y_{\min }[5,6]$. According to Weyl's Theorem [13, Theorem 4.3.1] for the Hermitian matrices $Q, Y_{\max }$ and the formulas (25) and (3) we write :

$$
\begin{aligned}
& \lambda_{k}(Q)-\lambda_{\max }\left(Y_{\max }\right) \leq \lambda_{k}\left(Q-Y_{\max }\right) \leq \lambda_{k}(Q)-\lambda_{\min }\left(Y_{\max }\right) \\
& \lambda_{k}(Q)-\lambda_{\max }\left(X_{\text {max }}^{s}\right) \leq \lambda_{k}\left(X_{\text {min }}^{s}\right) \leq \lambda_{k}(Q)-\lambda_{\text {min }}\left(X_{\text {max }}^{s}\right) \\
& \lambda_{k}(Q)-\lambda_{\text {max }}^{s}\left(X_{\max }\right) \leq \lambda_{k}^{s}\left(X_{\min }\right) \leq \lambda_{k}(Q)-\lambda_{\text {min }}^{s}\left(X_{\max }\right)
\end{aligned}
$$

Setting in (28) $k=\max$, due to $X \leq X_{\max } \leq Q^{1 / s} \quad[12$, Theorem 2.1], it is obvious that $\lambda_{k}(Q)-\lambda_{\text {max }}^{s}\left(X_{\max }\right) \geq 0$, thus (26) arises immediately. The validity of (27) is based on hypothesis and (28) for $k=\min$.

Moreover, it is well known $[1,4]$ that, when $A \in M_{n}$ is nonsingular, the existence of a finite number of Hermitian solutions of the matrix equation (4) depends on the eigenvalues of the matrix $\Phi$. In the following, $V\left(\lambda_{i}(\Phi)\right)$ denotes the eigenspace corresponding to the eigenvalue $\lambda_{i}(\Phi)$ and $\operatorname{dim}\left(V\left(\lambda_{i}(\Phi)\right)\right)$ denotes its dimension.

Theorem 9 Let $A \in M_{n}$ be a nonsingular matrix, $Q \in M_{n}$ with $Q>0$, and $\Phi$ be the matrix in (19) and assume that the matrix equation $X^{s}+A^{*} X^{-s} A=Q$ has at least one positive definite solution. If $\operatorname{dim}\left(V\left(\lambda_{i}(\Phi)\right)\right)=1$ for every eigenvalue $\lambda_{i}(\Phi), \quad i=1,2, \ldots, 2 n$, with $\left|\lambda_{i}(\Phi)\right| \neq 1$, then there exists a finite number of positive definite solutions (h.p.d.s.) of (1). Their number is equal to

\#h.p.d.s. $=\prod_{j=1}^{m}\left(n_{j}+1\right)$;

if $A, Q$ are real matrices, then among the h.p.d.s. there exist real symmetric solutions (r.p.d.s.) of (1) with 
$\#$ r.p.d.s. $=\prod_{k=1}^{p+q}\left(n_{k}+1\right)$

where $m$ is the number of the distinct eigenvalues of $\Phi$, that lie outside the unit circle, with algebraic multiplicity $n_{j}, \quad j=1,2, \ldots, m, \quad p$ is the number of real distinct eigenvalues of $\Phi$ lying outside the unit circle, $q$ is the number of complex conjugate pairs of eigenvalues lying outside the unit circle, with algebraic multiplicity $n_{k}$, $k=1,2, \ldots, p+q$.

Proof. Assume that $X \in M_{n}$ is a positive definite solution of (1); according to Theorem 7 every positive definite solution $X$ of (1) is related to a unique positive definite solution $Y \in M_{n}$ of (4) by (15). Hence, the unique symplectic matrix $\Phi$ in (19) corresponds to the two matrix equations (4) and (1), and consequently the total number of Hermitian (real symmetric) positive solutions of (1) is the same as in [4, Theorem 9] and given by (29)-(30).

\section{NUMERICAL RESULT}

In this section, we implement the proposed method to solve the matrix equation (1) and illustrate the main conclusions of the present paper. The proposed method computes the positive definite solutions using Matlab 6.5.

Example 1 Let the matrices

$A=\left[\begin{array}{cc}0.5 & 0.2 i \\ -0.3 & 0.4\end{array}\right], \quad Q=\left[\begin{array}{cc}1.2 & -0.3 \\ -0.3 & 2.1\end{array}\right]$

in the equation $X^{s}+A^{*} X^{-s} A=Q, \quad s \geq 1$. Obviously, $A$ is a nonsingular matrix, with

$\sigma(A)=\left\{\lambda_{1}(A)=0.2731+0.1696 i, \lambda_{2}(A)=0.6269-0.1696 i\right\}$, and $\left|\lambda_{1}(A)\right|=0.3215,\left|\lambda_{2}(A)\right|=0.6494$, while $Q$ is a positive definite matrix with $\sigma(Q)=\{1.1092,2.1908\}$.

Since $r\left(Q^{-1 / 2} A Q^{-1 / 2}\right)=0.4371$, Theorem 2 guarantees the existence of positive definite solutions of (1), hence the inequality (6) of Theorem 3 is verified. The spectrum of matrix $\Phi$ in (19) is

$$
\begin{aligned}
& \sigma(\Phi)=\left\{\lambda_{1}(\Phi)=-13.7403+16.3306 i\right. \\
& \lambda_{2}(\Phi)=-2.9676-0.8804 i, \\
& \lambda_{3}(\Phi)=-0.3097-0.0919 i, \\
&\left.\lambda_{4}(\Phi)=-0.0302+0.0359 i\right\}
\end{aligned}
$$

with

$$
\begin{aligned}
& \left|\lambda_{1}(\Phi)\right|=21.3421,\left|\lambda_{2}(\Phi)\right|=3.0954, \\
& \left|\lambda_{3}(\Phi)\right|=0.3231,\left|\lambda_{4}(\Phi)\right|=0.0469,
\end{aligned}
$$

thus $\Phi$ has $m=2$ eigenvalues outside the unit circle, the algebraic multiplicity of which is $n_{1}=n_{2}=1$. According to Theorem 9 the Riccati Equation Solution Method can be applied, because its assumptions are verified; the number of solutions is computed by (29) \#h.p.d.s. $=\prod_{j=1}^{m}\left(n_{j}+1\right)=4$.

The corresponding eigenvectors of $\Phi$ are the columns of the matrix

$W=\left[\begin{array}{cccc}-0.0030-0.2982 i & 0.6443 & 0.8989 & 0.6064-0.1393 i \\ 0.3927-0.0398 i & -0.1241-0.1102 i & 0.0449-0.2869 i & 0.7793 \\ -0.1105-0.3081 i & 0.6056+0.0438 i & 0.2843+0.0464 i & 0.0674-0.0108 i \\ 0.8051 & -0.4118-0.1382 i & -0.1322-0.0851 i & 0.0293-0.0037 i\end{array}\right]$.

The positive definite solutions of (4) are computed by (22); according to Theorem 7 and (15) the corresponding positive definite solutions of (1) are derived :

$$
\begin{aligned}
& Y_{1}=Y_{\text {min }}=\left[\begin{array}{cc}
0.3108 & -0.1554+0.0417 i \\
-0.1554-0.0417 i & 0.1659
\end{array}\right], \\
& U_{1}=\left[\begin{array}{cc}
0.8398 & 0.5243-0.1408 i \\
-0.5243-0.1408 i & 0.8398
\end{array}\right], \\
& D_{1}=\operatorname{diag}\{0.4148,0.0619\},
\end{aligned}
$$$$
X_{1}=X_{\text {min }}=U_{1}\left[\begin{array}{cc}
0.4148^{1 / s} & 0 \\
0 & 0.0619^{1 / s}
\end{array}\right] U_{1}^{*} \text {, }
$$

(minimal solution)

$$
\begin{aligned}
& Y_{2}=\left[\begin{array}{cc}
0.8123 & -0.5456+0.1314 i \\
-0.5456-0.1314 i & 0.4857
\end{array}\right], \\
& U_{2}=\left[\begin{array}{cc}
0.7998 & 0.5836-0.1405 i \\
-0.5836-0.1405 i & 0.7998
\end{array}\right], \\
& D_{2}=\operatorname{diag}\{1.2335,0.0645\},
\end{aligned}
$$$$
X_{2}=U_{2}\left[\begin{array}{cc}
1.2335^{1 / s} & 0 \\
0 & 0.0645^{1 / s}
\end{array}\right] U_{2}^{*}
$$$$
Y_{3}=\left[\begin{array}{cc}
0.4715 & -0.2323-0.45 i \\
-0.2323+0.45 i & 1.7066
\end{array}\right] \text {, }
$$$$
U_{3}=\left[\begin{array}{cc}
0.9416 & -0.1544-0.2992 i \\
0.1544-0.2992 i & 0.9416
\end{array}\right] \text {, }
$$$$
D_{3}=\operatorname{diag}\{0.2905,1.8877\} \text {, }
$$

$$
\begin{gathered}
X_{3}=U_{3}\left[\begin{array}{cc}
0.2905^{1 / s} & 0 \\
0 & 1.8877^{1 / s}
\end{array}\right] U_{3}^{*} \\
Y_{4}=Y_{\max }=\left[\begin{array}{cc}
0.9099 & -0.2623-0.1202 i \\
-0.2623+0.1202 i & 1.9568
\end{array}\right],
\end{gathered}
$$$$
U_{4}=\left[\begin{array}{cc}
0.9684 & -0.2266-0.1038 i \\
0.2266-0.1038 i & 0.9684
\end{array}\right] \text {, }
$$$$
D_{4}=\operatorname{diag}\{0.8356,2.0310\} \text {, }
$$

$X_{4}=X_{\max }=U_{4}\left[\begin{array}{cc}0.8356^{1 / s} & 0 \\ 0 & 2.0310^{1 / s}\end{array}\right] U_{4}^{*}$,

(maximal solution) 
It is also verified that: $0<X_{\min }<X_{\max }$.

\section{CONCLUSIONS}

In this paper, we investigate the positive definite solutions of the nonlinear matrix equation $X^{s}+A^{*} X^{-s} A=Q$. We derive necessary and sufficient conditions for the existence of the positive definite solutions, which are related to the theory and properties of the numerical radius of $Q^{-1 / 2} A Q^{-1 / 2}$. Some properties of the solutions are discussed as well as necessary and sufficient conditions for the existence of the positive definite solutions for special matrices $A, Q$ are derived. An algebraic method for computing all the positive definite solutions is proposed, which is based on the algebraic solution of the corresponding discrete time Riccati equation and the exact number of the positive definite solutions is found. The method provides simple formula for computing the solutions, as verified through a numerical result.

\section{REFERENCES}

Adam M, Assimakis N, Sanida F. Algebraic solutions of the matrix equations $X+A^{T} X^{-1} A=Q$ and $X-A^{T} X^{-1} A=Q$. Int J Algebra Comput 2008; 2(11): 501-18.

[2] Engwerda JC. On the existence of a positive definite solution of the matrix equation $X+A^{T} X^{-1} A=I$. Linear Algebra Appl 1993; 194: 91-108.

[3] Zhan X. Computing the extremal positive definite solutions of a matrix equation. SIAM J Sci Comput 1996; 17(5): 1167-74.

[4] Adam M, Assimakis N, Tziallas G, Sanida F. Riccati equation solution method for the computation of the solutions of $X+A^{T} X^{-1} A=Q$ and $X-A^{T} X^{-1} A=Q$. Open Appl Inform $\mathrm{J}$ 2009; 3: 22-33.
Engwerda JC, Ran ACM, Rijkeboer AL. Necessary and sufficient conditions for the existence of a positive definite solution of the matrix equation $X+A^{*} X^{-1} A=Q$. Linear Algebra Appl 1993; 186: 255-75.

[6] Meini B. Efficient computation of the extreme solutions of $X+A^{*} X^{-1} A=Q$ and $X-A^{*} X^{-1} A=Q$. Math Comput 2001; 71(239): 1189-204.

[7] Cai J, Chen G. Some investigation on Hermitian positive definite solutions of the matrix equation $X^{s}+A^{*} X^{-t} A=Q$. Linear Algebra Appl 2009; 430: 2448-56.

[8] Duan X, Liao A. On the existence of Hermitian positive definite solutions of the matrix equation $X^{s}+A^{*} X^{-t} A=Q$. Linear Algebra Appl 2008; 429: 673-87.

[9] Liu XG, Gao H. On the positive definite solutions of the matrix equations $X^{s} \pm A^{T} X^{-t} A=I_{n}$. Linear Algebra Appl 2003; 368: 8397.

[10] Cai J, Chen G. On the Hermitian positive definite solutions of nonlinear matrix equation $X^{s}+A^{*} X^{-t} A=Q$. Appl Math Comput 2010; 217: 117-23.

[11] Yin X, Liu S, Fanga L. Solutions and perturbation estimates for the matrix equation $X^{s}+A^{*} X^{-t} A=Q$. Linear Algebra Appl 2009; 431(9): 1409-21.

[12] Yueting Y. The iterative method for solving nonlinear matrix equation $X^{s}+A^{*} X^{-t} A=Q$. Appl Math Comput 2007; 188: 46-53.

[13] Horn RA, Johnson CR. Matrix analysis, $1^{s t}$ ed. Cambridge: Cambridge University Press 1985.

[14] Horn RA, Johnson CR. Topics in matrix analysis, $1^{\text {st }}$ ed. Cambridge: Cambridge University Press 1991; p. 607.

[15] Vaughan DR. A nonrecursive algebraic solution for the discrete riccati equation. IEEE Trans Automat Contr 1970; 597-99. 